\title{
Magnetotransport in disordered graphene exposed to ozone: From weak to strong localization
}

\author{
J. Moser, ${ }^{1}$ H. Tao, ${ }^{1, *}$ S. Roche,${ }^{1,2}$ F. Alzina, ${ }^{1}$ C. M. Sotomayor Torres, ${ }^{1,3}$ and A. Bachtold ${ }^{1}$ \\ ${ }^{1}$ CIN2 (CSIC-ICN) Barcelona, Campus UAB, E-08193 Bellaterra, Spain \\ ${ }^{2}$ INAC, SP2M, L_sim, CEA, 17 avenue des Martyrs, 38054 Grenoble, France \\ ${ }^{3}$ ICREA, 08010 Barcelona, Spain
}

(Received 5 March 2010; revised manuscript received 6 May 2010; published 28 May 2010)

\begin{abstract}
We present a magnetotransport study of graphene samples into which a mild disorder was introduced by exposure to ozone. Unlike the conductivity of pristine graphene, the conductivity of graphene samples exposed to ozone becomes very sensitive to temperature: it decreases by more than three orders of magnitude between 100 and $1 \mathrm{~K}$. By varying either an external gate voltage or temperature, we continuously tune the transport properties from the weak to the strong localization regime. We show that the transition occurs as the phase coherence length becomes comparable to the localization length. We also highlight the important role of disorder-enhanced electron-electron interaction on the resistivity.
\end{abstract}

DOI: 10.1103/PhysRevB.81.205445

PACS number(s): 72.80.Vp, 72.15.Rn

\section{INTRODUCTION}

Quantum interference phenomena in graphene are of fundamental interest. ${ }^{1}$ A case in point is the localization of charges, which is a manifestation of two important properties of this material: first, graphene hosts chiral Dirac fermions. Second, these fermions reside in two inequivalent valleys at the $\mathrm{K}$ and $\mathrm{K}^{\prime}$ points of the first Brillouin zone. Traveling paths that are relevant to weak-localization (WL) phenomena are phase coherent closed loops. Because of its chirality, a Dirac fermion residing in a given valley acquires a phase of $\pi$ upon completion of one loop, which gives rise to destructive interference with its time-reversed counterpart. Chirality therefore lowers the probability for returning paths and favors weak antilocalization. Restoring constructive interferences requires intervalley scattering events (fermions in the $\mathrm{K}$ and $\mathrm{K}^{\prime}$ valleys have opposite chiralities). This in turn favors weak localization.

These quantum interference effects have been actively studied both theoretically ${ }^{2-7}$ and experimentally. ${ }^{8-12}$ It has been predicted that the weak-localization correction to the semiclassical (Drude) conductivity will dominate the weakantilocalization correction as the temperature is lowered, driving graphene to the strong localization (SL) regime. ${ }^{3}$ Surprisingly however, these corrections measured in graphene samples have remained modest, even at millikelvin temperatures.

A clear strategy to study the transition between weak localization and strong localization is to enhance intervalley scattering. This can be achieved by introducing short-range scatterers, such as weak point disorder or lattice defects that result in midgap states. ${ }^{13-15}$ Recently, defect scattering centers were introduced in graphene using $\mathrm{Ne}$ and $\mathrm{He}$ ion irradiation but the conductivity at the Dirac point remained above $e^{2} / h$ even down to cryogenic temperature ${ }^{16}\left(e^{2} / h\right.$ is the conductivity value for which the weak-localization regime is expected to cross over to the strong localization one).

Approaches to drive the metallic phase of graphene to an insulator with an energy band gap have also been explored. In the case of graphane, ${ }^{17} s p^{2}$ bonds were partially trans- formed into $s p^{3}$ by hydrogenation. There, the resistivity was found to diverge at low temperature, in accordance with the two-dimensional variable-range hopping model. The measurements were interpreted as the result of a modified graphene that consists of two phases, regions with $s p^{3}$ hybridization interspersed with $s p^{2}$ regions. ${ }^{17}$ Similar results were obtained with oxidized graphene. ${ }^{18,19}$ Another work reported transport measurements deep in the strong localization regime using graphene modified with hydrogen atoms. ${ }^{20}$

Overall, these transport studies on intentionally disordered graphene focused either on the metallic regime ${ }^{16}$ or on the deep localization regime, where the material behaves as an insulator. ${ }^{17-20}$ The possible transition between weak localization and strong localization remains to be explored.

In this work, we report on a detailed magnetotransport study unveiling quantum localization effects in disordered graphene. Disorder is created by exposing graphene to ozone, which introduces $s p^{3}$-type defects. By varying either an external gate voltage or temperature, we continuously tune the transport properties from the weak localization to the strong localization regime. We show that the transition occurs as the phase coherence length becomes comparable to the localization length. In addition, we show evidence for a marked contribution of electron-electron interaction to the resistivity. (This latter effect is a correction to the density of states and its origin differs from the one of weak localization, which stems from a modification of the diffusion constant. ${ }^{21}$ )

\section{EXPERIMENTAL RESULTS}

Before creating defects in graphene, we first fabricate high-quality devices using conventional nanofabrication techniques (Fig. 1). ${ }^{22}$ We mechanically exfoliate graphene from a flake of Kish graphite on a Si wafer coated with 300 $\mathrm{nm}$ of thermal oxide. We pattern $\mathrm{Cr} / \mathrm{Au}$ electrodes in a fourpoint configuration using electron-beam lithography. We carry out Raman spectroscopy ${ }^{23}$ and low-temperature transport measurements to verify that single-layer graphene sheets are of good quality. For the device discussed in the paper, the $\mathrm{D}$ peak is absent before ozone treatment (Fig. 2, upper 


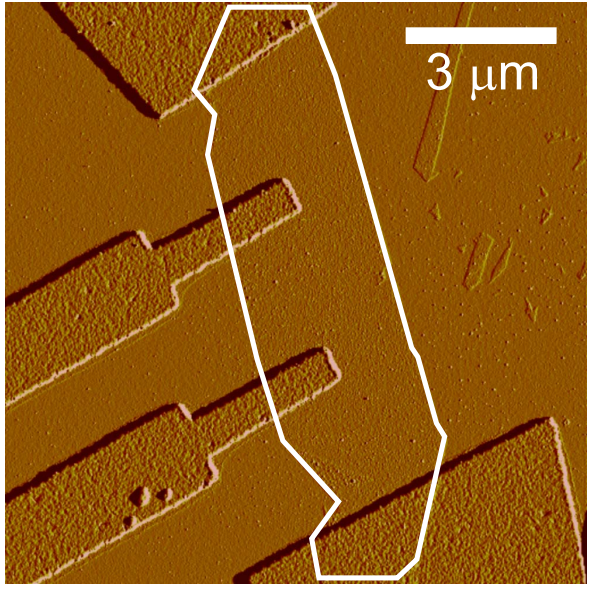

FIG. 1. (Color online) Atomic force microscopy image of the device contacted with four electrodes. The white contour highlights the sample shape.

panel). In addition, the mobility is $5500 \mathrm{~cm}^{2} / \mathrm{V} \mathrm{s}$, and the conductivity at the Dirac point reaches $6 e^{2} / h$ and is temperature independent down to liquid-helium temperature [Fig. 3(a)].

We introduce defect using an ozone treatment, which is a chemically reactive process known to alter the underlying $s p^{2}$ network of graphitic systems. Ozone molecules $\left(\mathrm{O}_{3}\right)$ are expected to preferentially form epoxide groups $(\mathrm{C}-\mathrm{O}-\mathrm{C})$ in graphene but other chemical groups can also be created such as $\mathrm{C}=\mathrm{C}, \mathrm{C}=\mathrm{O}$, and $\mathrm{C}-\mathrm{O} .^{24}$ Specifically, we first clean graphene samples by placing them in a flow of $\mathrm{Ar} / \mathrm{H}_{2}$ gas at $300{ }^{\circ} \mathrm{C}$ for $3 \mathrm{~h}$. We then expose the samples to ozone in a Novascan ozone chamber, where ozone is produced by ultraviolet irradiation of $\mathrm{O}_{2}$ gas $(\sim 7 \mathrm{~min}, 4 \mathrm{~atm})$. The appearance of the D peak in the Raman spectrum (Fig. 2, lower panel) and the decrease in the mobility down to $390 \mathrm{~cm}^{2} / \mathrm{V} \mathrm{s}$ (see below) signal the creation of additional defects.

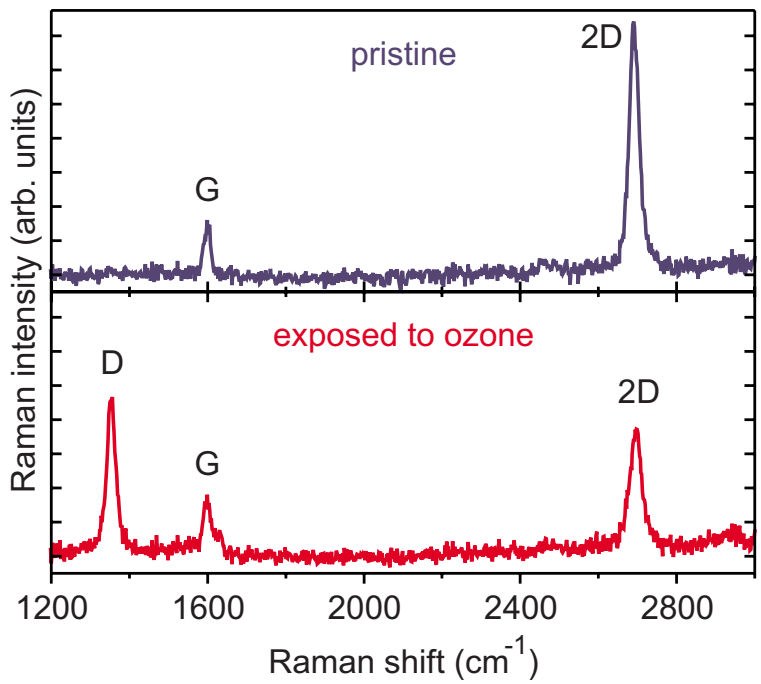

FIG. 2. (Color online) Raman spectrum of the device shown in Fig. 1 before (top) and after (bottom) exposure to ozone. G and 2D modes are shown; defects created by ozone reveal themselves as a strong D mode. Both panels have the same intensity axis.
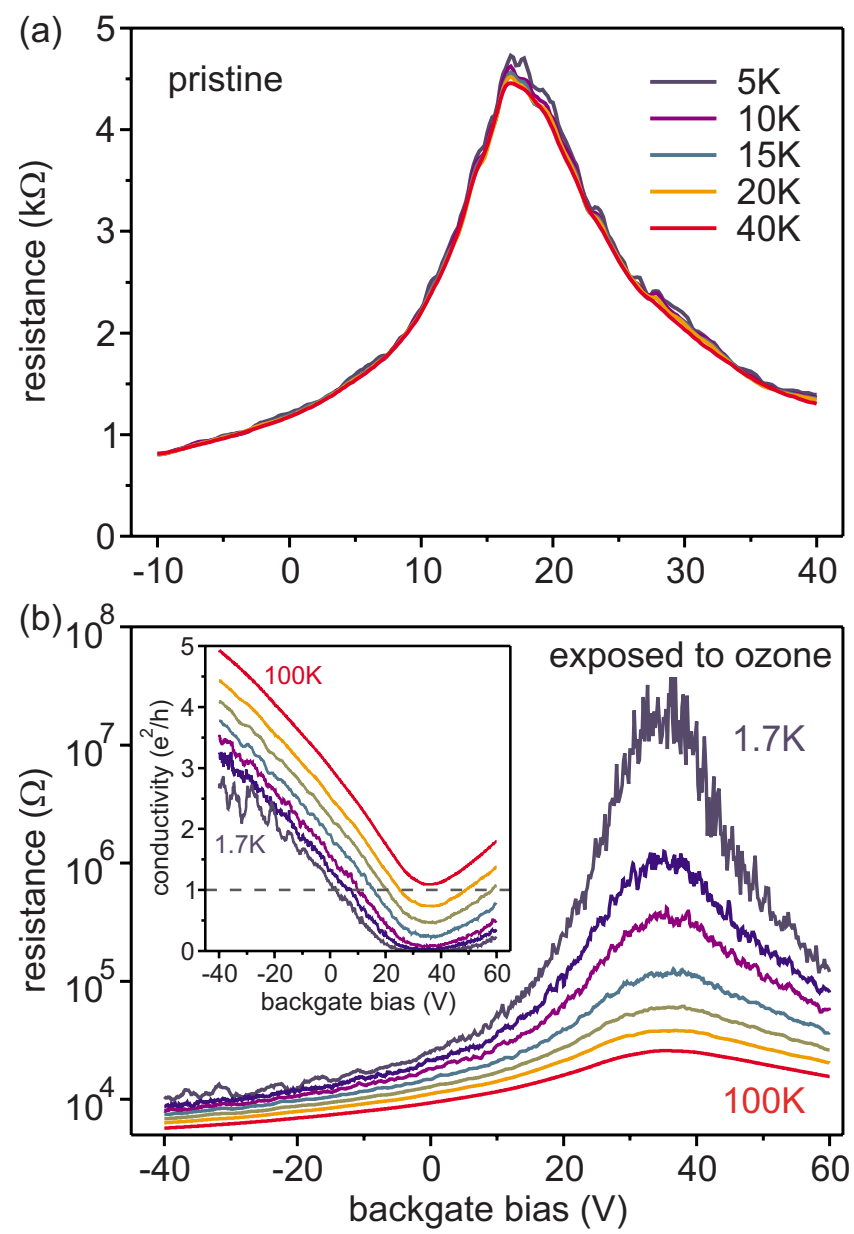

FIG. 3. (Color online) Four-point resistance of the sample as a function of backgate bias $V_{g}$ (a) before and (b) after ozone exposure. In (b), temperatures are $T=1.7,3,5,10,20,40$, and $100 \mathrm{~K}$. Inset to panel (b) displays the corresponding four-point conductivity as a function of backgate bias $V_{g}$.

We, however, make sure that the ozone treatment is mild enough to preserve the crystalline integrity of graphene. Indeed, the elastic mean-free path of electrons estimated from the Drude conductivity is at least $3 \mathrm{~nm}$ (see below), which is more than one order of magnitude larger than the $\mathrm{C}-\mathrm{C}$ bond length. The presence of the $\mathrm{G}$ peak and a well-defined 2D peak in Raman spectroscopy further support the $s p^{2}$ nature of the material, the ratio of the integrated $D$ and $G$ band intensities $I_{D}$ and $I_{G}$ yielding a characteristic length of disorder $L=6 \mathrm{~nm}$ [using the phenomenological relation $L(\mathrm{~nm})$ $=2.4 \times 10^{-10}\left(\mathrm{~nm}^{-3}\right) \cdot \lambda^{4}\left(\mathrm{~nm}^{4}\right) \cdot I_{G} / I_{D}, 25,26 \quad$ with $\lambda$ $=514.5 \mathrm{~nm}]$, which is rather close to the elastic mean-free path obtained from the transport measurements.

This ozone treatment has a large impact on the transport properties of graphene. The conductivity $\sigma$ becomes very sensitive to temperature $T$ [Fig. 3(b)]. Even though the conductivity at the Dirac point remains larger than $e^{2} / h$ at the highest temperature $(100 \mathrm{~K})$, it is reduced by more than three orders of magnitude at $1.7 \mathrm{~K}$. The device behaves as an insulator, at least at low temperature and in the vicinity of the Dirac point.

To reveal the contributions of quantum interferences, we explore the transport properties in the presence of a magnetic 

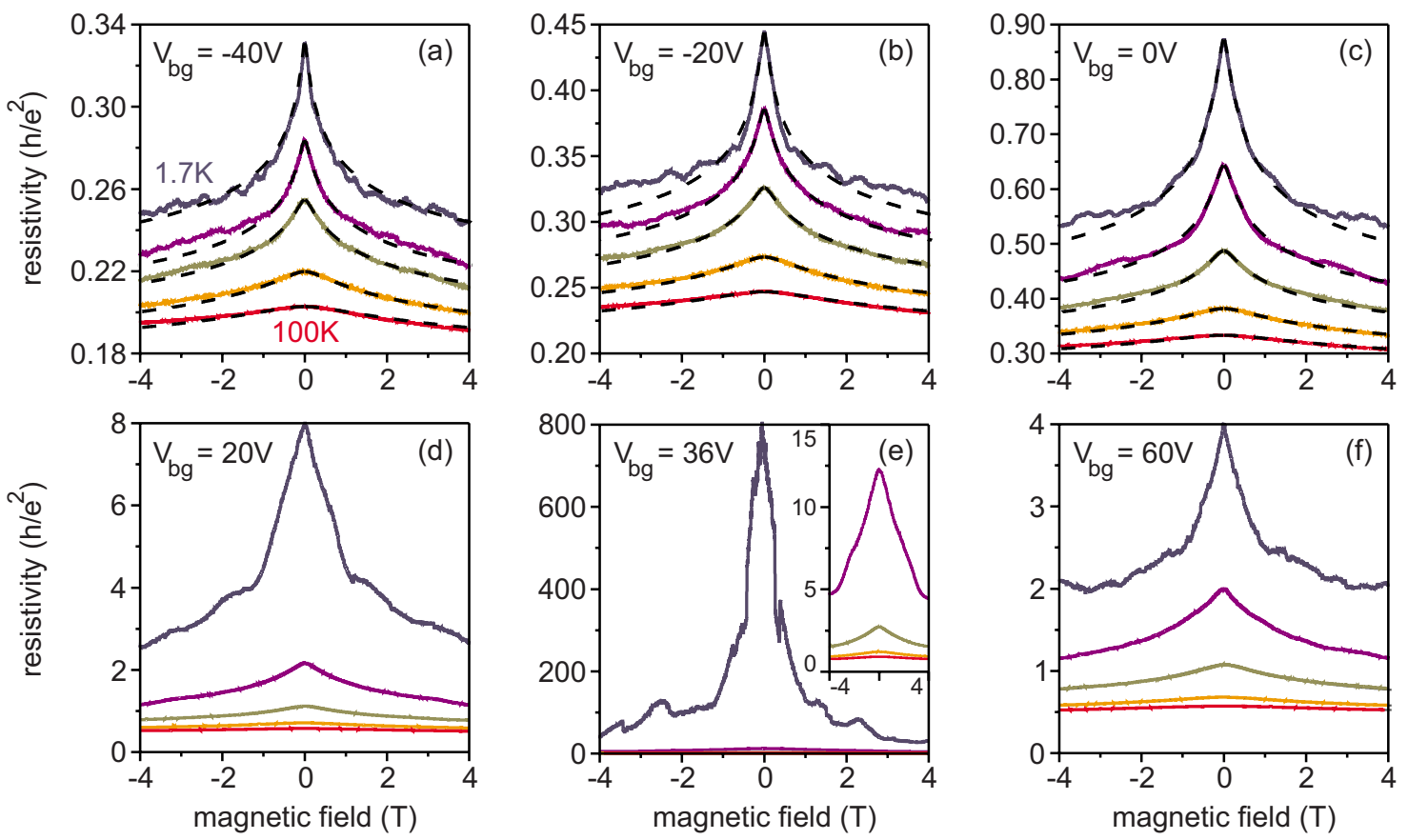

FIG. 4. (Color online) Resistivity as a function of magnetic field, for $T=1.7,5,15,50$, and $100 \mathrm{~K}$. Fits to Eq. (1) are shown by dashed lines away from the Dirac point and for conductivity values larger than $e^{2} / h$. Inset of Fig. 4(e) displays the resistivity at the Dirac point at $5,15,50$, and $100 \mathrm{~K}$ (top to bottom).

field $B$ [Figs. 4(a)-4(f)]. In all cases, the magnetoresistance is negative, and the resistivity changes with $B$ by an amount that strongly depends on $V_{g}$. This change becomes increasingly large as the density approaches the Dirac point $\left[V_{g}\right.$ $=36 \mathrm{~V}$, Fig. 4(e)] whereas it remains moderate at high charge density $n\left[V_{g}=-40 \mathrm{~V}\right.$, Fig. $\left.4(\mathrm{a})\right]$.

\section{DISCUSSION}

Both the low-temperature insulating behavior ${ }^{3,5}$ and the sharp D peak in Raman spectra show that the ozone treatment introduces significant intervalley scattering. A way to estimate the intervalley as well as the intravalley scattering rates is to examine the $V_{g}$ dependence of the conductivity. Assuming only weak point disorder and charged impurity disorder, we obtain that the intervalley and the intravalley scattering times are comparable, about 10 fs for $V_{g}=-40 \mathrm{~V}$. Conversely, before ozone treatment the intervalley scattering time and the intravalley scattering time are both about $150 \mathrm{fs}$ at the same $n$. To determine these scattering times, we consider weak point disorder for the intervalley scattering time $\tau_{\text {inter }}=\sigma_{\mathrm{sr}} \cdot h /\left(2 e^{2} \cdot v_{F} \sqrt{\pi n}\right)$ and charged-impurity disorder for the intravalley scattering time $\tau_{\text {intra }}=h /\left(2 e \cdot v_{F} \sqrt{\pi}\right) \cdot \mu \cdot \sqrt{n}$. The conductivity $\sigma_{\mathrm{sr}}$ (due to short-range scattering) and the mobility $\mu$ can be extracted from the hole branch of the $\sigma\left(V_{g}\right)$ measurement taken at $100 \mathrm{~K}$ (assuming that localization effects are vanishingly small at higher temperature) using $\sigma^{-1}=\sigma_{\mathrm{sr}}^{-1}+(e \mu n)^{-1} .^{27} \mathrm{We}$ get $\sigma_{\mathrm{sr}}=5 \times 10^{-3} \mathrm{~S}$ and $\mu$ $=5500 \mathrm{~cm}^{2} / \mathrm{V} \mathrm{s}$ for the pristine graphene, and $\sigma_{\mathrm{sr}}=4.1$ $\times 10^{-4} \mathrm{~S}$ and $\mu=390 \mathrm{~cm}^{2} / \mathrm{V} \mathrm{s}$ after ozone treatment. We note that lattice defects resulting in midgap states were recently identified as a new source of intervalley scattering. ${ }^{16}$
The latter scattering results in a linear $n$ dependence of $\sigma$ so its contribution to the conductivity cannot be discriminated from the one of charged-impurity disorder in a $\sigma\left(V_{g}\right)$ measurement. As such, it can modify $\tau_{\text {inter }}$ and $\tau_{\text {intra }}$ and the intervalley scattering time of $10 \mathrm{fs}$ is an upper bound.

The magnetoresistance measurements at high $n$ can be well described by the WL theory developed for graphene. ${ }^{6}$ The correction to the semiclassical (Drude) conductivity reads

$$
\begin{aligned}
\delta \sigma_{\text {graphene }}= & \frac{e^{2}}{\pi h}\left[F\left(\frac{\tau_{B}^{-1}}{\tau_{\phi}^{-1}}\right)-F\left(\frac{\tau_{B}^{-1}}{\tau_{\phi}^{-1}+2 \tau_{\text {inter }}^{-1}}\right)\right. \\
& \left.-2 F\left(\frac{\tau_{B}^{-1}}{\tau_{\phi}^{-1}+\tau_{\text {inter }}^{-1}+\tau_{\text {intra }}^{-1}}\right)\right]
\end{aligned}
$$

with $F(z)=\ln z+\psi\left(0.5+z^{-1}\right)$, where $\psi$ is the digamma function, $\tau_{B}^{-1}=4 e D B / \hbar$, and $\tau_{\phi}=L_{\phi}^{2} / D$ the phase coherence time. To compare with the experiment, we let the diffusion constant $D=0.5 v_{F}^{2} \cdot\left(\tau_{\text {inter }}^{-1}+\tau_{\text {intra }}^{-1}\right)^{-1}$ and consider only weak point disorder for intervalley scattering and charged-impurity disorder for intravalley scattering. Accordingly, the phase coherence time $\tau_{\phi}$ is the only fitting parameter necessary. As illustrated in Figs. 4(a) $-4(\mathrm{c})$ [where $\sigma(B=0)>e^{2} / h$ ], we find a good agreement between experiments and theory. A satisfactory agreement is also obtained by comparing measurements to WL predictions for conventional two-dimensional metals, which, moreover, yields the same phase coherence time. As for the magnetoresistance measurements at lower $n$ [Figs. 4(d) $-4(\mathrm{f})$ ], the resistivity can change with $B$ by a large amount. Comparing the measurements to theory is however difficult at this stage. More measurements at low temperature 
(a)

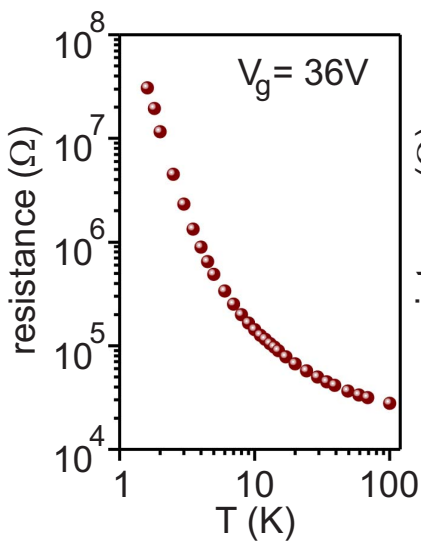

(b)

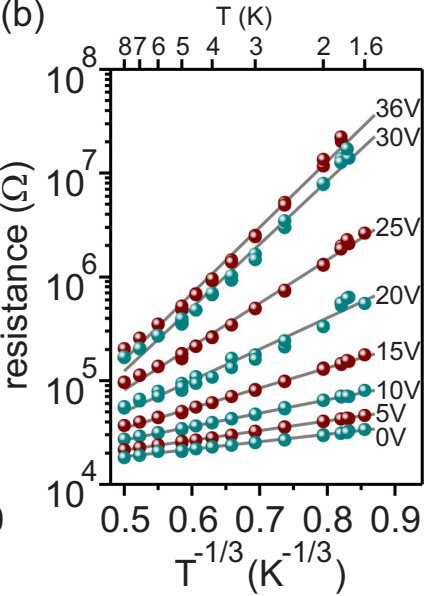

FIG. 5. (Color online) (a) Temperature dependence of the resistance at the Dirac point. (b) At low temperature, the sample resistance is fit to the two-dimensional variable-range hopping model for several gate biases in the vicinity of the Dirac point.

will be needed to discern among the various predicted dependencies. ${ }^{28}$

We now turn our attention to the temperature dependence of the resistance. The resistance at the Dirac point diverges at low temperature, as expected for an insulating regime [Fig. 5(a)]. Figure 5(b) shows that the temperature-dependent resistance is consistent with two-dimensional variable-range hopping, $R \propto \exp \left[\left(T_{0} / T\right)^{1 / 3}\right]$. Even though the measurement could also be described by a simple thermal activation behavior, for the time being we will restrict our analysis to a variable-range hopping scenario, which is the conventional mechanism to describe low-temperature conduction in strongly disordered materials. ${ }^{17-19,29}$ This allows us to extract the localization length $\xi_{V R H}$ from the fitting parameter $T_{0},{ }^{29}$

$$
\xi_{\text {VRH }}=\sqrt{\frac{13.8}{k_{B} \rho T_{0}}},
$$

where $\rho$ is the density of states of graphene. To estimate $\rho$, we assume that $n=C_{g} \sqrt{\Delta V_{g}^{2}+V_{0}^{2}} / e$ to take the fluctuations of the Dirac point with respect to the Fermi energy into account $\left(\Delta V_{g}\right.$ is the backgate bias measured from the conductivity minimum). Here, $C_{g}=1.15 \times 10^{-4} \mathrm{~F} / \mathrm{m}^{2}$ is the backgate capacitance. ${ }^{30}$ Letting $V_{0}=8 \mathrm{~V}$ (which roughly accounts for the smoothing of the $\sigma$ vs $V_{g}$ curve around the Dirac point at the highest temperature), we obtain $\xi_{V R H}=15 \mathrm{~nm}$ at the Dirac point. Away from the Dirac point, we find that $\xi_{V R H}$ increases upon increasing $n$ [Fig. 6(a)]. As a comparison, we can evaluate the localization length using the rough estimate, ${ }^{31}$

$$
\xi_{D} \simeq L_{e} \exp \left(\frac{\sigma_{\mathrm{D}}}{e^{2} / h}\right) .
$$

The elastic length $L_{e}$ is derived from the Drude conductivity $\sigma_{\mathrm{D}}$ as $L_{e}=\sigma_{\mathrm{D}} \cdot h /\left(2 e^{2} \sqrt{\pi n}\right)$, assuming that $\sigma_{\mathrm{D}}$ is the conductivity measured at a temperature of $100 \mathrm{~K}$. This yields $\xi_{D}$ $\simeq 12 \mathrm{~nm}$ at the Dirac point, which is close to the variablerange hopping estimate. The agreement is also quite reason- (a)

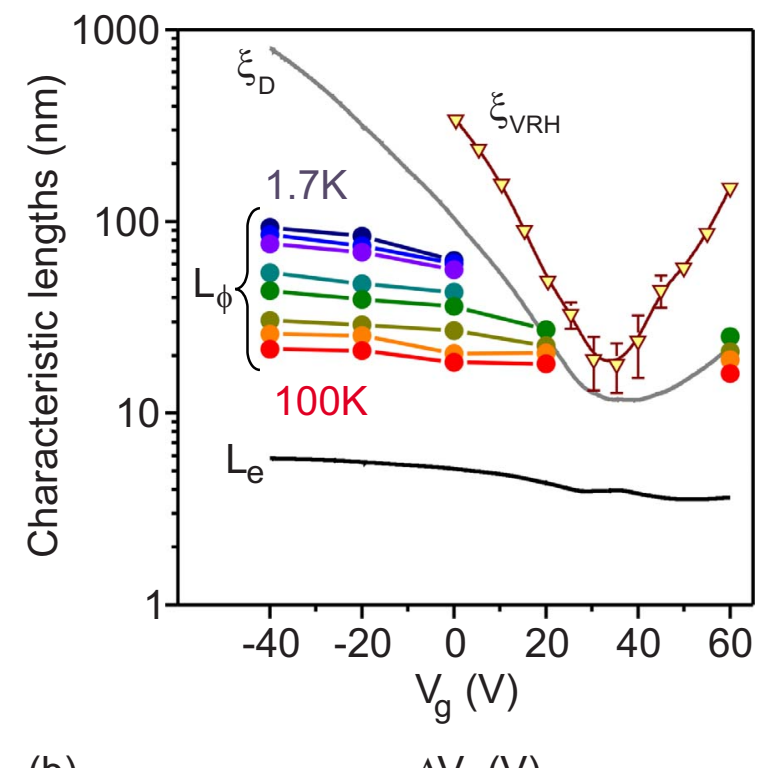

(b)

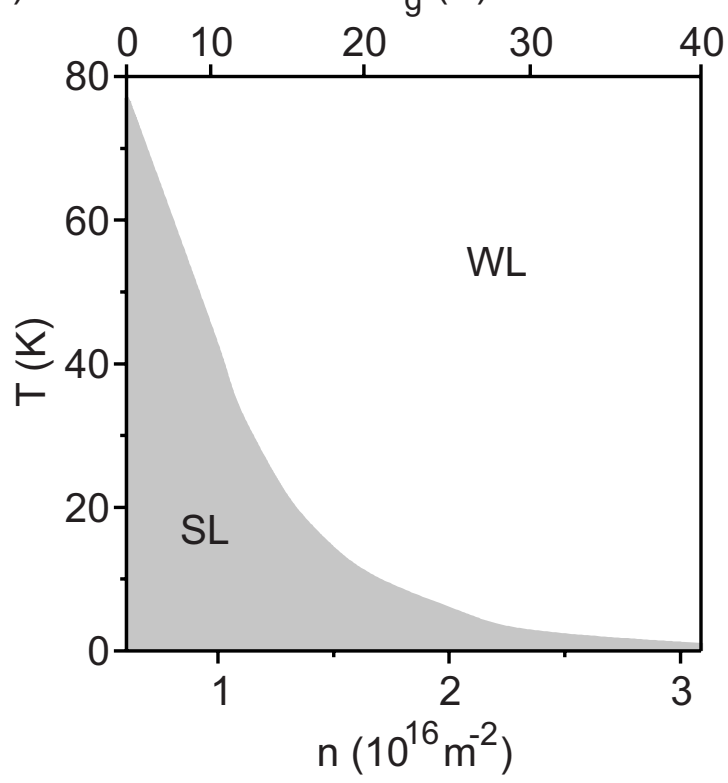

FIG. 6. (Color online) (a) Characteristic lengths as a function of backgate bias $V_{g} . L_{e}$ is the elastic mean-free path. Coherence lengths $L_{\phi}$ at $T=1.7,3,5,15,25,50,70$, and $100 \mathrm{~K}$ (top to bottom) are shown. $\xi_{V R H}$ is the localization length estimated from the variable-range hopping model [Eq. (2)] and $\xi_{D}$ is the localization length estimated from the scaling theory [Eq. (3)]. (b) Phase diagram (temperature $T$ and charge density $n$ ) showing the semimetallic regime where WL is observed and the insulator regime where SL prevails. The top axis shows the corresponding backgate biases $\Delta V_{g}$ measured from the Dirac point.

able at higher $n$ [Fig. 6(a)]. Overall, this rough agreement gives us confidence in the order of magnitude of the localization length.

The transition from weak localization to strong localization can be understood by contrasting the fundamental transport length scales extracted from our experimental data [Fig. 6(a)]. As long as the phase coherence length $L_{\phi}$ remains 
smaller than the estimated localization length $\xi$, the weaklocalization regime prevails. Whenever $L_{\phi}$ becomes comparable to $\xi$, we observe that the conductivity is close to $e^{2} / h$, the value at which the transition to strong localization is expected. ${ }^{32}$ Figure 6(a) displays $L_{\phi}$ only when $\sigma>e^{2} / h$ at zero magnetic field (and except at the Dirac point). In the opposite case $\left(\sigma<e^{2} / h\right)$, the comparison between experiment and weak-localization theory becomes worse, so that extracting a value for $L_{\phi}$ is meaningless.

Graphene offers the possibility to tune the carrier density $n$ with $V_{g}$, which provides a practical knob to test the localization theory. From our measurements, we can construct a "phase diagram" of the transition from WL to SL as a function of $n$ and temperature [Fig. 6(b)]. To do this, we use the temperature dependence of $\sigma$ [a few such curves are shown in the inset of Fig. 3(b)] and define the transition as $\sigma$ $=e^{2} / h$. We emphasize that the transition is expected to be gradual and to develop as a smooth crossover. ${ }^{32}$ Figure 6(b) shows that the WL-SL transition is very sensitive to $n$ at low carrier concentration. This is because of the strong variation in $\xi$ upon varying $n$ [Fig. 6(a)]. By contrast, the other fundamental transport lengths, $L_{e}$ and $L_{\phi}$, remain essentially constant.

Eventually, one interesting outcome of our measurements is that they reveal the importance of Coulomb interaction between charge carriers. This can be seen in the magnetoresistance measurements at high magnetic field [Fig. 4(a)] where the conductivity at $B=4 \mathrm{~T}$ is definitely temperature dependent. The contribution of weak localization is reduced at high magnetic field and the remaining correction to $\sigma$ is usually attributed to Coulomb interaction. The correction to the conductivity due to Coulomb interaction in a twodimensional metal reads ${ }^{21,33}$

$$
\delta \sigma_{e e}=-\frac{e^{2}}{2 \pi^{2} \hbar} \cdot g_{2 \mathrm{D}} \cdot \ln \left(\frac{\hbar}{k_{B} T \tau_{e}}\right),
$$

where $g_{2 \mathrm{D}}$ is a constant on the order of unity. Figure 7 shows that the experiment agrees well with the theory using $g_{2 \mathrm{D}}$ $=0.8$. This Coulomb interaction effect and weak localization have different origins. ${ }^{21}$ Weak localization arises from quantum interference between electronic paths and modifies the diffusion constant, whereas disorder-induced electronelectron interaction originates from quantum correlation between electrons and it modifies the density of states. These results indicate that the correction to the conductivity due to Coulomb interaction cannot be neglected in the transition between weak localization and strong localization in graphene, as it is treated in existing theoretical works. ${ }^{2-7,34-36}$

\section{CONCLUSION}

We have reported on the crossover from weak localization to strong localization in disordered graphene, the disorder being created with ozone. For this, we have carried out magnetotransport measurements as a function of gate voltage and

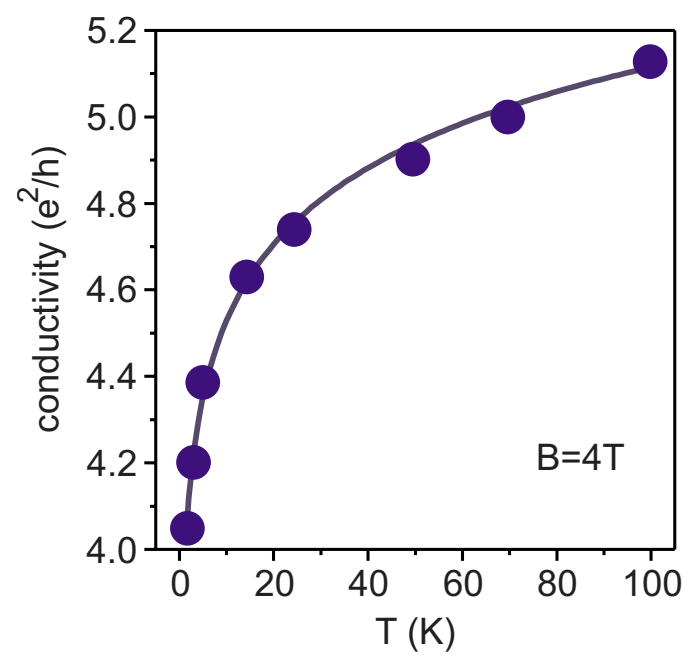

FIG. 7. (Color online) Temperature dependence of the conductivity at $V_{g}=-40 \mathrm{~V}$ and $B=4 \mathrm{~T}$ (dots), and fit to Eq. (4) (solid line).

temperature. We have shown that the transition between weak and strong localization occurs as the phase coherence length becomes comparable to the localization length and that the transition is very sensitive to the charge-carrier concentration. In addition, we have demonstrated the importance of the resistivity correction due to disorder-induced electronelectron interaction.

Previous works showed that disorder in graphene obtained by hydrogenation or oxidation can open an energy gap. ${ }^{17-19}$ By contrast, recent calculations show that this is not the case for graphene exposed to ozone. ${ }^{37}$ In addition, even if such a gap was created, our measurements suggest that it would be very small. Indeed, we obtain an energy gap of 1 meV when we compare the temperature dependence of the resistivity at the Dirac point to a thermal activation behavior. This low value suggests that a gap, if it exists, would be relevant only to measurements near the Dirac point (in our case, it would be included in the interpretation of the measurements at $\left.V_{g}=36 \mathrm{~V}\right)$. Further experimental work is needed to demonstrate whether this energy gap exists or not using, e.g., scanning tunneling microscopy techniques. Moreover, measurements will be carried out to characterize the microscopic modifications brought by exposing graphene to ozone, such as the chemical nature and the distribution of defects.

\section{ACKNOWLEDGMENTS}

We are grateful to M. Lira-Cantu for the use of the ozone chamber and acknowledge fruitful discussion with F. Guinea. This work was supported by a EURYI grant, the EU under Grant No. FP6-IST-021285-2, and the MICINN under Grants No. FIS2008-06830 and No. FIS2009-10150. S.R. acknowledges the ANR/P3N2009 (NANOSIM-GRAPHENE Project No. ANR-09-NANO-016-01). 
* Present address: Research Institute of Micro/Nano Science and Technology, Shanghai Jiao Tong University, 200240 Shanghai, China.

${ }^{1}$ E. McCann, Phys. 2, 98 (2009).

${ }^{2}$ H. Suzuura and T. Ando, Phys. Rev. Lett. 89, 266603 (2002).

${ }^{3}$ I. L. Aleiner and K. B. Efetov, Phys. Rev. Lett. 97, 236801 (2006).

${ }^{4}$ A. Altland, Phys. Rev. Lett. 97, 236802 (2006).

${ }^{5}$ P. M. Ostrovsky, I. V. Gornyi, and A. D. Mirlin, Phys. Rev. B 74, 235443 (2006).

${ }^{6}$ E. McCann, K. Kechedzhi, V. I. Fal'ko, H. Suzuura, T. Ando, and B. L. Altshuler, Phys. Rev. Lett. 97, 146805 (2006).

${ }^{7}$ A. F. Morpurgo and F. Guinea, Phys. Rev. Lett. 97, 196804 (2006).

${ }^{8}$ S. V. Morozov, K. S. Novoselov, M. I. Katsnelson, F. Schedin, L. A. Ponomarenko, D. Jiang, and A. K. Geim, Phys. Rev. Lett. 97, 016801 (2006).

${ }^{9}$ X. Wu, X. Li, Z. Song, C. Berger, and W. A. de Heer, Phys. Rev. Lett. 98, 136801 (2007).

${ }^{10}$ F. V. Tikhonenko, D. W. Horsell, R. V. Gorbachev, and A. K. Savchenko, Phys. Rev. Lett. 100, 056802 (2008).

${ }^{11}$ F. V. Tikhonenko, A. A. Kozikov, A. K. Savchenko, and R. V. Gorbachev, Phys. Rev. Lett. 103, 226801 (2009).

${ }^{12}$ Y.-F. Chen, M.-H. Bae, C. Chialvo, T. Dirks, A. Bezryadin, and N. Mason, J. Phys.: Condens. Matter 22, 205301 (2010).

${ }^{13}$ T. Ando, J. Phys. Soc. Jpn. 75, 074716 (2006).

${ }^{14}$ E. H. Hwang, S. Adam, and S. Das Sarma, Phys. Rev. Lett. 98, 186806 (2007).

${ }^{15}$ T. Stauber, N. M. R. Peres, and F. Guinea, Phys. Rev. B 76, 205423 (2007).

${ }^{16}$ J.-H. Chen, W. G. Cullen, C. Jang, M. S. Fuhrer, and E. D. Williams, Phys. Rev. Lett. 102, 236805 (2009).

${ }^{17}$ D. C. Elias, R. R. Nair, T. M. G. Mohiuddin, S. V. Morozov, P. Blake, M. P. Halsall, A. C. Ferrari, D. W. Boukhvalov, M. I. Katsnelson, A. K. Geim, and K. S. Novoselov, Science 323, 610 (2009).

${ }^{18}$ C. Gómez-Navarro, R. T. Weitz, A. M. Bittner, M. Scolari, A. Mews, M. Burghard, and K. Kern, Nano Lett. 7, 3499 (2007).

${ }^{19}$ A. B. Kaiser, C. Gómez-Navarro, R. S. Sundaram, M. Burghard, and K. Kern, Nano Lett. 9, 1787 (2009).

${ }^{20}$ A. Bostwick, J. L. McChesney, K. V. Emtsev, T. Seyller, K. Horn, S. D. Kevan, and E. Rotenberg, Phys. Rev. Lett. 103, 056404 (2009).

${ }^{21}$ C. W. J. Beenakker and H. van Houten, Solid State Phys. 44, 1 (1991).

${ }^{22}$ J. Moser, A. Barreiro, and A. Bachtold, Appl. Phys. Lett. 91,
163513 (2007)

${ }^{23}$ A. C. Ferrari, J. C. Meyer, V. Scardaci, C. Casiraghi, M. Lazzeri, F. Mauri, S. Piscanec, D. Jiang, K. S. Novoselov, S. Roth, and A. K. Geim, Phys. Rev. Lett. 97, 187401 (2006).

${ }^{24}$ G. Lee, B. Lee, J. Kim, and K. Cho, J. Phys. Chem. C 113, 14225 (2009).

${ }^{25}$ L. G. Cançado, K. Takai, T. Enoki, M. Endo, Y. A. Kim, H. Mizusaki, A. Jorio, L. N. Coelho, R. Magalhaes-Paniago, and M. A. Pimenta, Appl. Phys. Lett. 88, 163106 (2006).

${ }^{26}$ A. C. Ferrari, Solid State Commun. 143, 47 (2007).

${ }^{27}$ C. Jang, S. Adam, J.-H. Chen, E. D. Williams, S. Das Sarma, and M. S. Fuhrer, Phys. Rev. Lett. 101, 146805 (2008).

${ }^{28}$ D. Yoshioka, Y. Ono, and H. Fukuyama, J. Phys. Soc. Jpn. 50, 3419 (1981); V. L. Nguyen, B. Z. Spivak, and B. I. Shklovskii, Sov. Phys. JETP 62, 1021 (1985); Y. Ono, Prog. Theor. Phys. Suppl. 84, 138 (1985); U. Sivan, O. Entin-Wohlman, and Y. Imry, Phys. Rev. Lett. 60, 1566 (1988); O. Entin-Wohlman, Y. Imry, and U. Sivan, Phys. Rev. B 40, 8342 (1989); B. I. Shklovskii and B. Z. Spivak, in Hopping Transport in Solids, edited by M. Pollak and B. I. Shklovskii (Elsevier, New York, 1991); H. L. Zhao, B. Z. Spivak, M. P. Gelfand, and S. Feng, Phys. Rev. B 44, 10760 (1991); P. Kleinert and V. V. Bryksin, ibid. 55, 1469 (1997).

${ }^{29}$ B. I. Shklovskii and A. L. Efros, Electronic Properties of Doped Semiconductors (Springer-Verlag, Berlin, 1984).

${ }^{30}$ A. Barreiro, M. Lazzeri, J. Moser, F. Mauri, and A. Bachtold, Phys. Rev. Lett. 103, 076601 (2009).

${ }^{31}$ M. E. Gershenson, Y. B. Khavin, A. G. Mikhalchuk, H. M. Bozler, and A. L. Bogdanov, Phys. Rev. Lett. 79, 725 (1997).

${ }^{32}$ P. A. Lee and T. V. Ramakrishnan, Rev. Mod. Phys. 57, 287 (1985).

${ }^{33}$ B. L. Altshuler, A. G. Aronov, and P. A. Lee, Phys. Rev. Lett. 44, 1288 (1980).

${ }^{34}$ K. Nomura, M. Koshino, and S. Ryu, Phys. Rev. Lett. 99, 146806 (2007); K.-I. Imura, Y. Kuramoto, and K. Nomura, EPL 89, 17009 (2010).

${ }^{35}$ J. P. Robinson, H. Schomerus, L. Oroszlány, and V. I. Fal'ko, Phys. Rev. Lett. 101, 196803 (2008).

${ }^{36}$ A. Lherbier, B. Biel, Y.-M. Niquet, and S. Roche, Phys. Rev. Lett. 100, 036803 (2008); A. Cresti, N. Nemec, B. Biel, G. Niebler, F. Triozon, G. Cuniberti, and S. Roche, Nano Res. 1, 361 (2008); A. Lherbier, X. Blase, Y.-M. Niquet, F. Triozon, and S. Roche, Phys. Rev. Lett. 101, 036808 (2008).

${ }^{37}$ N. Leconte, J. Moser, P. Ordejon, H. Tao, A. Lherbier, A. Bachtold, F. Alsina, C. M. Sotomayor-Torres, J.-C. Charlier, and S. Roche, ACS Nano (to be published). 